\title{
Loss of Tuberous Sclerosis Complex1 in Adult Oligodendrocyte Progenitor Cells Enhances Axon Remyelination and Increases Myelin Thickness after a Focal Demyelination
}

\author{
는 \\ and Teresa L. Wood ${ }^{1}$ \\ ${ }^{1}$ Department of Pharmacology, Physiology, and Neuroscience and Cancer Center, New Jersey Medical School, Rutgers University, Newark, New Jersey \\ 07101, and 2Department of Cell and Developmental Biology, University of Colorado School of Medicine, Aurora, Colorado 80045
}

\begin{abstract}
Although the mammalian target of rapamycin (mTOR) is an essential regulator of developmental oligodendrocyte differentiation and myelination, oligodendrocyte-specific deletion of tuberous sclerosis complex (TSC), a major upstream inhibitor of mTOR, surprisingly also leads to hypomyelination during CNS development. However, the function of TSC has not been studied in the context of remyelination. Here, we used the inducible Cre-lox system to study the function of TSC in the remyelination of a focal, lysolecithin-demyelinated lesion in adult male mice. Using two different mouse models in which $T s c 1$ is deleted by Cre expression in oligodendrocyte progenitor cells (OPCs) or in premyelinating oligodendrocytes, we reveal that deletion of $T s c 1$ affects oligodendroglia differently depending on the stage of the oligodendrocyte lineage. $T s c 1$ deletion from $\mathrm{NG}^{+}{ }^{+} \mathrm{OPCs}$ accelerated remyelination. Conversely, $T s c 1$ deletion from proteolipid protein (PLP)-positive oligodendrocytes slowed remyelination. Contrary to developmental myelination, there were no changes in OPC or oligodendrocyte numbers in either model. Our findings reveal a complex role for TSC in oligodendrocytes during remyelination in which the timing of Tscl deletion is a critical determinant of its effect on remyelination. Moreover, our findings suggest that TSC has different functions in developmental myelination and remyelination.
\end{abstract}

Key words: adult OPCs; mTOR; NG2; remyelination; TSC

\section{Significance Statement}

Myelin loss in demyelinating disorders such as multiple sclerosis results in disability due to loss of axon conductance and axon damage. Encouragingly, the nervous system is capable of spontaneous remyelination, but this regenerative process often fails. Many chronically demyelinated lesions have oligodendrocyte progenitor cells (OPCs) within their borders. It is thus of great interest to elucidate mechanisms by which we might enhance endogenous remyelination. Here, we provide evidence that deletion of Tscl from OPCs, but not differentiating oligodendrocytes, is beneficial to remyelination. This finding contrasts with the loss of oligodendroglia and hypomyelination seen with $T s c 1$ or $T s c 2$ deletion in the oligodendrocyte lineage during CNS development and points to important differences in the regulation of developmental myelination and remyelination.

\section{Introduction}

Within the CNS, oligodendrocytes are tasked with the myelination of axons to facilitate rapid signal conduction and provide

\footnotetext{
Received Nov. 3, 2016; revised June 9, 2017; accepted June 17, 2017.

Author contributions: L.E.M. and T.L.W. designed research; L.E.M., J.N.B., A.V.E., and L.K. performed research; W.B.M. contributed unpublished reagents/analytic tools; L.E.M. and T.L.W. analyzed data; L.E.M. and T.L.W. wrote the paper.

This work was supported by National Institute of Neurological Disorders and Stroke-National Institutes of Health (Grant NS082203 to W.B.M. and T.L.W.), the National Multiple Sclerosis Society (Grant RG5371-A-4), and the Department of Defense Tuberous Sclerosis Complex Research Program (Exploration Award TS093091 to T.L.W.). We thank Quan Shang and Danielle Harlow for technical assistance, Sharyl Fyffe-Maricich for helpful discussions, and Amy Davidow for statistical consultation and analysis.
}

trophic and metabolic support (Griffiths et al., 1998; LappeSiefke et al., 2003). Loss of myelin and oligodendrocytes in the adult CNS is a hallmark of demyelinating diseases such as multiple sclerosis (MS). Spontaneous endogenous remyelination occurs initially in some MS lesions, but often fails after repeated

\footnotetext{
The authors declare no competing financial interests.
}

Correspondence should be addressed to Teresa L. Wood, Ph.D., Department Pharmacology, Physiology, and Neuroscience, New Jersey Medical School Cancer Center H1200, Rutgers University, 205 S. Orange Ave, Newark, NJ 07101-1709. E-mail: Terri.wood@rutgers.edu.

DOI:10.1523/JNEUROSCI.3454-16.2017

Copyright $\odot 2017$ the authors $\quad 0270-6474 / 17 / 377534-13 \$ 15.00 / 0$ 
demyelinating attacks (Patrikios et al., 2006). Moreover, the myelin formed during remyelination is characteristically thinner than that formed during development and the remyelination capacity of the CNS diminishes with age (Shields et al., 1999; Goldschmidt et al., 2009). Therefore, much research has focused on elucidating the mechanisms underlying oligodendroglia maturation and myelination in both the developmental and the remyelination context to develop therapies to better promote remyelination (Keough and Yong, 2013).

The mammalian target of rapamycin (mTOR), and particularly mTOR complex 1 (mTORC1), has emerged as a key modulator of developmental oligodendrocyte maturation and myelination. Disrupting $\mathrm{mTORC} 1$ signaling perinatally in mice through the deletion of mTOR or the mTORC1 adaptor protein raptor results in delayed oligodendrocyte differentiation and hypomyelination (Bercury et al., 2014; Lebrun-Julien et al., 2014; Wahl et al., 2014; Zou et al., 2014). A similar phenotype was observed after deleting the small GTPase Rheb, a direct upstream activator of mTORC1 (Zou et al., 2014). Conversely, enhancing mTOR signaling during development is sufficient to increase myelin thickness: either deletion of the upstream inhibitor phosphatase and tensin homolog (PTEN) or constitutive activation of AKT results in increased mTOR signaling and hypermyelination (Flores et al., 2008; Harrington et al., 2010). Moreover, the hypermyelination phenotype due to constitutive AKT activation is mTOR dependent because it can be inhibited by rapamycin, a pharmacological inhibitor of mTOR (Narayanan et al., 2009).

The tuberous sclerosis complex (TSC), consisting of hamartin (TSC1) and tuberin (TSC2), acts upstream of Rheb to inhibit mTORC1 signaling (van Slegtenhorst et al., 1998; Inoki et al., 2003; Zhang et al., 2003). Given the evidence that mTOR signaling is a positive regulator of developmental myelination, inactivation of TSC might be expected to cause hypermyelination similar to the phenotype resulting from PTEN deletion or expression of constitutively activated AKT. It is thus surprising that several studies recently reported that deletion of $T s c 1$ or $T s c 2$ in oligodendrocyte progenitor cells (OPCs) during development in mouse models results in hypomyelination (Lebrun-Julien et al., 2014; Carson et al., 2015; Jiang et al., 2016). Disruption to the oligodendrocyte lineage was not explicitly examined when $T s c 1$ was deleted using CNP-Cre, but mRNA expression of oligodendroglia transcription factors Olig2 and Sox10 were reduced, suggesting fewer cells in the lineage (Lebrun-Julien et al., 2014). Jiang et al. (2016) deleted Tsc1 using Olig1-Cre and showed increased oligodendrocyte apoptosis attributed to elevated endoplasmic reticulum (ER) stress. In contrast, Carson et al. (2015) demonstrated a fate shift from the oligodendroglial to the astrocyte lineage after deleting $T s c 2$ using an Olig2-Cre.

The phosphoinositide 3-kinase (PI3K)/AKT/mTOR signaling pathway has not been studied extensively in remyelination. Systemic administration of the pharmacological mTOR inhibitor rapamycin inhibited remyelination in mice fed a cuprizone diet (Sachs et al., 2014). Surprisingly, deletion of PTEN from the oligodendroglial lineage during development had no effect on remyelination of a focal demyelinated lesion in the adult spinal cord, although the brains and spinal cords of these mice were already hypermyelinated due to developmental effects (Harrington et al., 2010).

The goal of the current study was to determine how deletion of $T s c 1$ from the oligodendrocyte lineage affects remyelination of a focal demyelinating insult in the adult spinal cord. To date, TSC deletion in oligodendrocyte precursors before developmental myelination has resulted in hypomyelination, the opposite of what one might expect from hyperactivating mTORC1 signaling.
Here, we demonstrate that deletion of $T s c 1$ from $\mathrm{NG}_{2}{ }^{+}$adult OPCs augments endogenous remyelination, resulting in more rapid remyelination, which is consistent with the classical concept of the role of TSC1 inhibition of mTORC1. Furthermore, we show that this beneficial effect is stage specific such that deletion of $T s c 1$ in differentiating proteolipid protein-positive $\left(\mathrm{PLP}^{+}\right)$ oligodendrocytes fails to improve remyelination.

\section{Materials and Methods}

Experimental animals. All mouse protocols were conducted in accordance with Rutgers University Institutional Animal Care and Use Committee. Mice homozygous for the floxed-Tsc1 allele (The Jackson Laboratory 005680, RRID:IMSR_JAX:005680) were bred with either NG2-Cre ${ }^{\text {ERT2 }}$ mice (The Jackson Laboratory 008538, RRID:IMSR_JAX: $008538)$ to generate NG2-Cre; $T s c 1^{\mathrm{fl} / \mathrm{fl}}$ mice, henceforth referred to as NG2-Tsc1 conditional knock-out (cKO) mice or with PLP-Cre ${ }^{\text {ERT2 }}$ mice (The Jackson Laboratory 005975, RRID:IMSR_JAX:005975) to generate PLP-Cre; Tsc $1^{\mathrm{fl} / \mathrm{fl}}$, henceforth referred to as PLP-Tsc1cKO mice. The resulting mice were on a mixed C57BL/6J, BALB/cJ, 129/SvJ background. The lines also carried a dual-reporter transgene (B6.129(Cg)Gt(ROSA)26Sortm4 (ACTB-tdTomato,-EGFP)Luo/J, Jackson Laboratories, RRID: IMSR_JAX:007676) that allowed for assessment of recombination efficiency and specificity as all cells express tomato red (mTmG) in the absence of active Cre-recombinase and green fluorescent protein (GFP) when Cre-recombinase is active. Only males were used in the remyelination experiments to reduce background recombination in females due to endogenous estrogen.

For remyelination studies, tamoxifen was injected intraperitoneally (75 $\mathrm{mg} / \mathrm{kg})$ to induce recombination in adult (8-12 weeks old) male mice. Tamoxifen was dissolved in a 9:1 ratio of sesame oil:100\% ethanol. NG2-Tsc1cKO mice were injected with tamoxifen for 5 consecutive days, followed by $4 \mathrm{~d}$ to clear the tamoxifen from the system before lysolecithin injections into the dorsal white matter (see below). Controls were either of the same genotype and injected with vehicle or were Cre negative (WT; $T s c 1^{\text {fl/fl }}$; dual-reporter mice) and injected with tamoxifen. Because these controls were not phenotypically distinct from each other, they were combined into a single control group in the subsequent analyses. PLPTsc1cKO or control Cre-negative mice were injected with tamoxifen for 6 consecutive days beginning $8 \mathrm{~d}$ after the lysolecithin injection. All controls were Cre-negative tamoxifen-injected animals to control for any effects of tamoxifen on remyelination.

To validate loss of TSC1 in the NG2-Tsc1cKO line, we injected tamoxifen $(75 \mathrm{mg} / \mathrm{kg})$ for 5 consecutive days into Cre-negative or Cre-positive mice at 4 weeks of age $(n=3$ per genotype). Animals were taken for tissue harvesting $18 \mathrm{~d}$ after the last tamoxifen injection to mimic the timing of the analyses in the lesioned animals. $\mathrm{NG} 2^{+}$cells were isolated from brains and spinal cords using NG2 microbeads (Miltenyi Biotec 130-097170) and MS columns (Miltenyi Biotec 130-042-201) and used for protein isolation. To validate TSC1 deletion in the PLP-Tsc1cKO line, Cre-positive or Cre-negative mice at PND $8(n=3$ per genotype) were injected with tamoxifen $(60 \mathrm{mg} / \mathrm{kg})$ for 4 consecutive days. Animals were taken for tissue harvesting $5 \mathrm{~d}$ after the last tamoxifen injection to test for loss of TSC1 to resemble the time frame for the paradigm used for the lesion analyses in the PLP-Tsc1cKO line, in which animals were killed $8 \mathrm{~d}$ after the final tamoxifen injection. The $\mathrm{O}^{+}{ }^{+}$oligodendrocytes were isolated from spinal cords using O4 microbeads (Miltenyi Biotec 130-094543) and MS columns (Miltenyi Biotec 130-042-201) and used for protein isolation. Total protein was isolated from either the $\mathrm{NG} 2{ }^{+}$or $\mathrm{O}^{+}{ }^{+}$cells from these two deletion paradigms using RIPA lysis buffer (Thermo Scientific 89900) containing protease and phosphatase inhibitors and separated by SDS-PAGE as described previously (Tyler et al., 2009). TSC1 antibody (Cell Signaling Technology 6935, RRID:AB_10860420) was diluted 1:1000 and incubated overnight at $4^{\circ} \mathrm{C}$.

Lysolecithin-induced demyelination. Lysolecithin (Sigma-Aldrich L4129) was injected into the dorsal column of the thoracic spinal cord of adult male mice 8-13 weeks of age. A dorsal laminectomy was performed at T10 of the spinal cord and a hole was made in the dura using a 26 gauge syringe needle. A $5 \mu$ l Neuros syringe (Hamilton 65460-02) with a custom 33 gauge 
needle (Hamilton 7803-05) was lowered into the dorsal white matter until the needle bevel was entirely within the cord. Then, $1 \mu \mathrm{l}$ of $1 \%$ lysolecithin dissolved in a $0.9 \% \mathrm{NaCl}$ solution was injected at a rate of $0.25-0.3 \mu \mathrm{l} / \mathrm{min}$. The needle was left in place for $2 \mathrm{~min}$ after the injection to prevent liquid reflux. The injection site was marked with charcoal for identification.

Tissue preparation and immunostaining. NG2-Tsc1cKO animals and corresponding controls were taken for analysis at $7 \mathrm{~d}$ postlesion $(\mathrm{dpl})$, when lesion size peaks; $14 \mathrm{dpl}$, a time point that corresponds to early remyelination; and $21 \mathrm{dpl}$, when more robust remyelination is present (Jeffery and Blakemore, 1995; Luo et al., 2014). Animals within the PLPTsc1cKO group and their corresponding controls were taken for analysis at $21 \mathrm{dpl}$. A total of three to six animals per genotype from the corresponding time point were used for all analyses described.

Mice were intracardially perfused with $10 \mathrm{ml}$ of an ice-cold PBS solution containing phosphatase inhibitors and heparin followed by $40 \mathrm{ml}$ of ice-cold $3 \%$ paraformaldehyde (PFA) at a rate of $2 \mathrm{ml} / \mathrm{min}$. Spinal cord tissue $2 \mathrm{~mm}$ from either side of the injection site was dissected and drop-fixed in 3\% PFA overnight. The tissue was then dehydrated with a $30 \%$ sucrose solution and subsequently frozen in OCT. Next, $12 \mu \mathrm{m}$ spinal cord cross-sections were taken throughout the lesion as defined by DAPI hypercellularity.

Mounted cryosections were rinsed in TBS, permeabilized in 1\% Triton $\mathrm{X}-100$ and $2 \%$ normal serum for $30 \mathrm{~min}$, and then blocked in a solution containing $10 \%$ BSA and $10 \%$ normal serum for $1 \mathrm{~h}$. Normal serum was from goat, rat, or donkey, depending on the species in which the secondary antibody was raised. Primary antibodies were incubated at $4^{\circ} \mathrm{C}$ overnight. Sections were washed in TBS with $0.05 \%$ Triton X-100 and incubated in secondary antibodies for $3 \mathrm{~h}$. Both primary and secondary antibodies were diluted in a TBS solution containing $2 \%$ BSA, $2 \%$ serum, and $0.2 \%$ Triton X-100. For CC1 and MBP staining, antigen retrieval was performed before permeabilization; sections were placed in a $10 \mathrm{~mm}$ solution of sodium citrate and microwaved $3 \times 40 \mathrm{~s}$ at medium power with $25 \mathrm{~min}$ in between microwaving sessions. For MBP staining, sections were first treated with $50 \mathrm{~mm}$ ammonium chloride to reduce autofluorescence and then delipidated with $100 \%$ ethanol for $10 \mathrm{~min}$. The M.O.M. kit (Vector Laboratories BMK-2202, RRID:AB_2336833) was used for CC1 staining. After permeabilization, slides were first incubated in M.O.M. mouse Ig blocking reagent for $1 \mathrm{~h}$ followed by a $30 \mathrm{~min}$ incubation with CC1 antibody in M.O.M. diluent and then standard blocking solution and other primary antibodies. The following primary antibodies were used: CC1 (1:100, Millipore OP80, RRID:AB_2057371), Cleaved Caspase 3 (1:500, Cell Signaling Technology 9661, RRID:AB_2341188), GFP (1:500, Aves Laboratories GFP-1020, RRID:AB_10000240), Ibal (1:750, Wako Technology 019-19741, RRID:AB_839504), MBP (1:1000, Covance SMI-99, RRID:AB_2314772), Olig2 (1:750, a gift from Dr. Charles Stiles, Harvard University, RRID:AB_2336878), PDGFR $\beta$ (1:200, Abcam ab32570, RRID: AB_777165; 1:200 ab91066, RRID:AB_10563302), phospho-S6 (1:750, Cell Signaling Technology 4858, RRID:AB_916156), Sox10 (1:25, R\&D Systems AF2864, AB_442208) and TSC1 (1:500, Bio-Rad AHP1001, RRID: AB_609656). Secondary antibodies were as follows: $\mathrm{G} \alpha \mathrm{M} 647$ (1:750, Invitrogen A21235, RRID:AB_2535804), G $\alpha$ R AMCA (1:200, Jackson Laboratories, 111-155-144, RRID:AB_2337994), G $\alpha$ Ck 488 (1:1000, Invitrogen A11039, RRID:AB_2534096), D $\alpha \mathrm{M} 647$ (1:750, Invitrogen A31571, RRID:AB_162542), D $\alpha$ Gt AMCA (1:200, Jackson Laboratories 705-155-147, RRID:AB_2340409), D $\alpha$ Ck 488 (1:700, Jackson Laboratories 703-545-155, RRID:AB_2340375), Rt $\alpha$ M IgG2 $\beta 647$ (1:350, Abcam ab172327, RRID:AB_2650513).

For myelin oligodendrocyte glycoprotein (MOG) immunohistochemistry, mounted cryosections were rinsed, delipidated with $100 \%$ ethanol for $10 \mathrm{~min}$, and then incubated in $3 \% \mathrm{H}_{2} \mathrm{O}_{2}$ to eliminate endogenous peroxidase activity. Sections were permeabilized in $0.3 \%$ Triton $\mathrm{X}-100$ and then blocked against nonspecific binding as described above. Sections were incubated with MOG primary antibody (1:1000, Abcam ab32760, RRID:AB_2145529) overnight followed by incubation in a biotinylated $\mathrm{G} \alpha \mathrm{Rb}$ secondary antibody (1:500, Vector Laboratories BA1000, RRID:AB_2313606) for $2 \mathrm{~h}$, streptavidin ( $1 \mu \mathrm{g} / \mathrm{ml}$, Pierce 21126) for an additional $2 \mathrm{~h}$. Positive signal was detected using the NovaRed substrate kit (Vector Laboratories SK-4800, RRID:AB_2336845). Sec- tions were dehydrated and coverslipped with Cytoseal (Thermo Scientific 8312-4).

Toluidine blue and electron microscopy (EM). Animals were intracardially perfused with $40 \mathrm{ml}$ of cold PBS followed by $40 \mathrm{ml}$ of modified Karnovsky's fixative (2\% paraformaldehyde $/ 2.5 \%$ glutaraldehyde) at a rate of $2 \mathrm{ml} / \mathrm{min}$. The spinal cord was removed and postfixed in the same fixative. The spinal cord was bisected through the middle of the lesion, embedded in $5 \%$ agarose in $0.1 \mathrm{~m}$ phosphate buffer, and sliced on a vibratome into 70 - to $200-\mu \mathrm{m}$-thick sections. Sections with clear lesions in the dorsal columns were postfixed in $1 \%$ osmium and $1.5 \%$ potassium ferrocyanide followed by $1 \%$ osmium tetroxide, dehydrated in graded acetone with 2\% uranyl acetate, and resin embedded in Embed 812 (Electron Microscopy Sciences 14120) using a PelcoBiowave Pro tissue processor (Ted Pella). One-micrometer-thick sections were cut from a trapezoid positioned over the dorsal columns and mounted onto glass slides where they were then stained with a mixture of $1 \%$ toluidine blue, $1 \%$ methylene blue, and $1 \%$ sodium borate in water for $\sim 1 \mathrm{~min}$. Ultrathin sections $(65 \mathrm{~nm})$ were cut at the same location within the lesion and mounted on Formvar-coated slot grids (EMS). Blocks were sectioned deeper into the lesion and another set of thick and ultrathin sections were collected for each sample. Ultrathin sections were viewed at $80 \mathrm{kV}$ on a Tecnai G2 transmission electron microscope (FEI) and imaged with a side-mount AMT XR80S-B camera.

Experimental design and statistical analysis. Mouse strains, experimental and control groups, and sex are detailed above in the sections on experimental animals and tissue preparation. Initial lesion size was determined by Luxol fast blue staining of sections $80 \mu \mathrm{m}$ apart throughout the lesion. The area of demyelination in a given segment of the lesion was measured within each section and the volume estimated using the cylindrical rule (volume $=$ area of selected section $\times$ distance between sections). Whole lesion volume was obtained by summing individual volumes. All immunofluorescence and MOG immunohistochemistry analyses were performed within the lesion borders, as defined by DAPI hypercellularity, on a minimum of 3 distinct sections, a minimum of 84 $\mu \mathrm{m}$ apart with $n=3$ animals per genotype. For MBP and MOG remyelination analyses, the area of the lesion that was immunopositive was measured in Image and expressed as a percentage of the total lesion area as defined by DAPI hypercellularity. For MBP demyelination analysis at $7 \mathrm{dpl}$, the MBP-negative area was measured in ImageJ from a crosssection at lesion peak. For immunohistochemistry analysis, the experimental and control groups were compared with a Student's $t$ test except for instances when multiple time points were being assessed, in which case an ANOVA was used to assess statistical significance.

For toluidine blue analysis, lesion borders were defined by the presence of darkly staining immune bodies and lack of organized axonal arrangement due to increased interstitial space. A minimum of two independent sections were analyzed for each animal. Three blinded, independent investigators ranked all cross-sections in a given set compared with one another. Rankings were based on the extent and density of remyelinated axons throughout the lesion, with a rank of 1 assigned to the cross-section with the least remyelination. To test for differences between remyelination rankings of the three raters, we used Friedman's $\chi^{2}$ test, a nonparametric analog of the randomized block ANOVA, and tested for block effects in which each rater was treated as a block (Daniel, 1990). The Friedman test was implemented using SAS proc survey freq following the approach of Ipe (1987). To test for the association between the extent of remyelination and group, a binary variable was created from the remyelination ranks using the mid-rank as the cutoff between high and low ranks. We then modeled group membership using a logbinomial regression model with the binary version of the ranks as the predictor variable, and controlling for multiple rankers using the generalized estimating approach (Zeger and Liang, 1986). We assumed an independent working correlation and the sandwich estimator was used to estimate SEs. Exponentiating coefficients of this model produced the relative rates of membership in the control group compared with the cKO group for specimens that were ranked highly.

Areas for EM analysis were selected from toluidine blue images from animals that ranked along the median of their respective groups. Selected areas were in similar places within the lesion in all animals: well within 


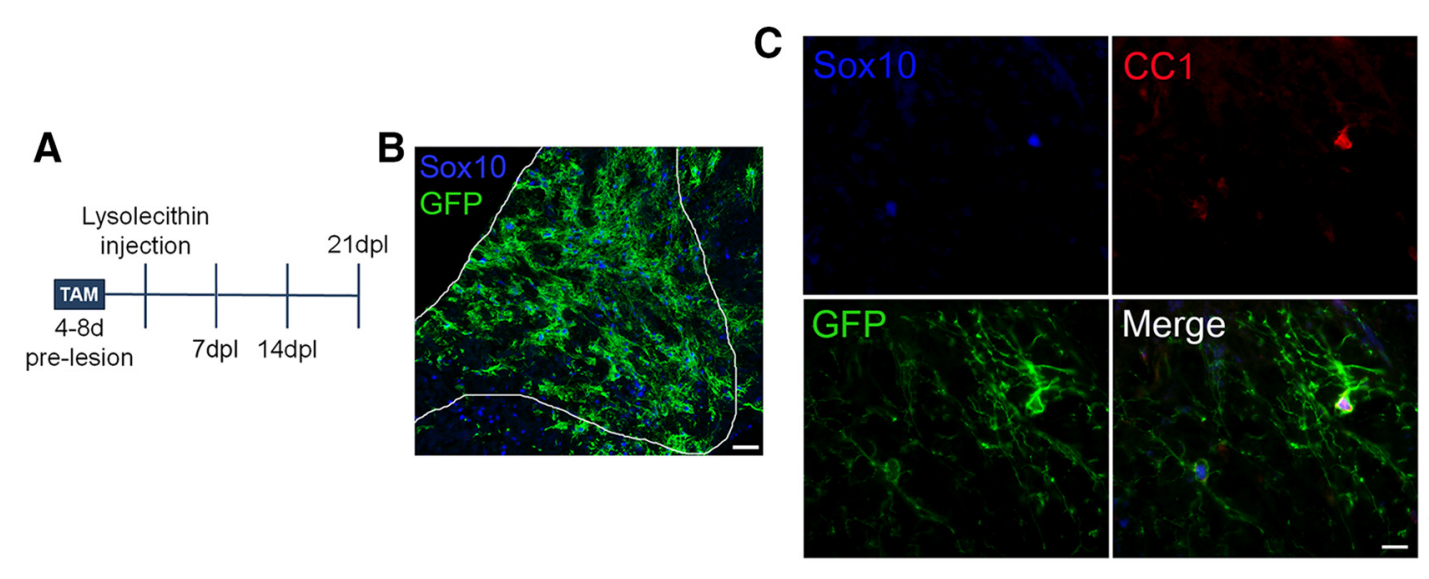

D
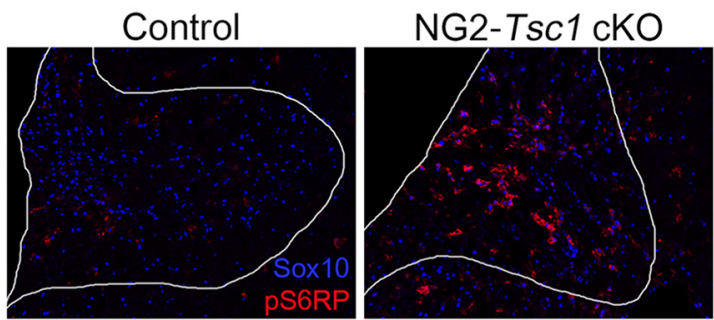

$\mathbf{E}$
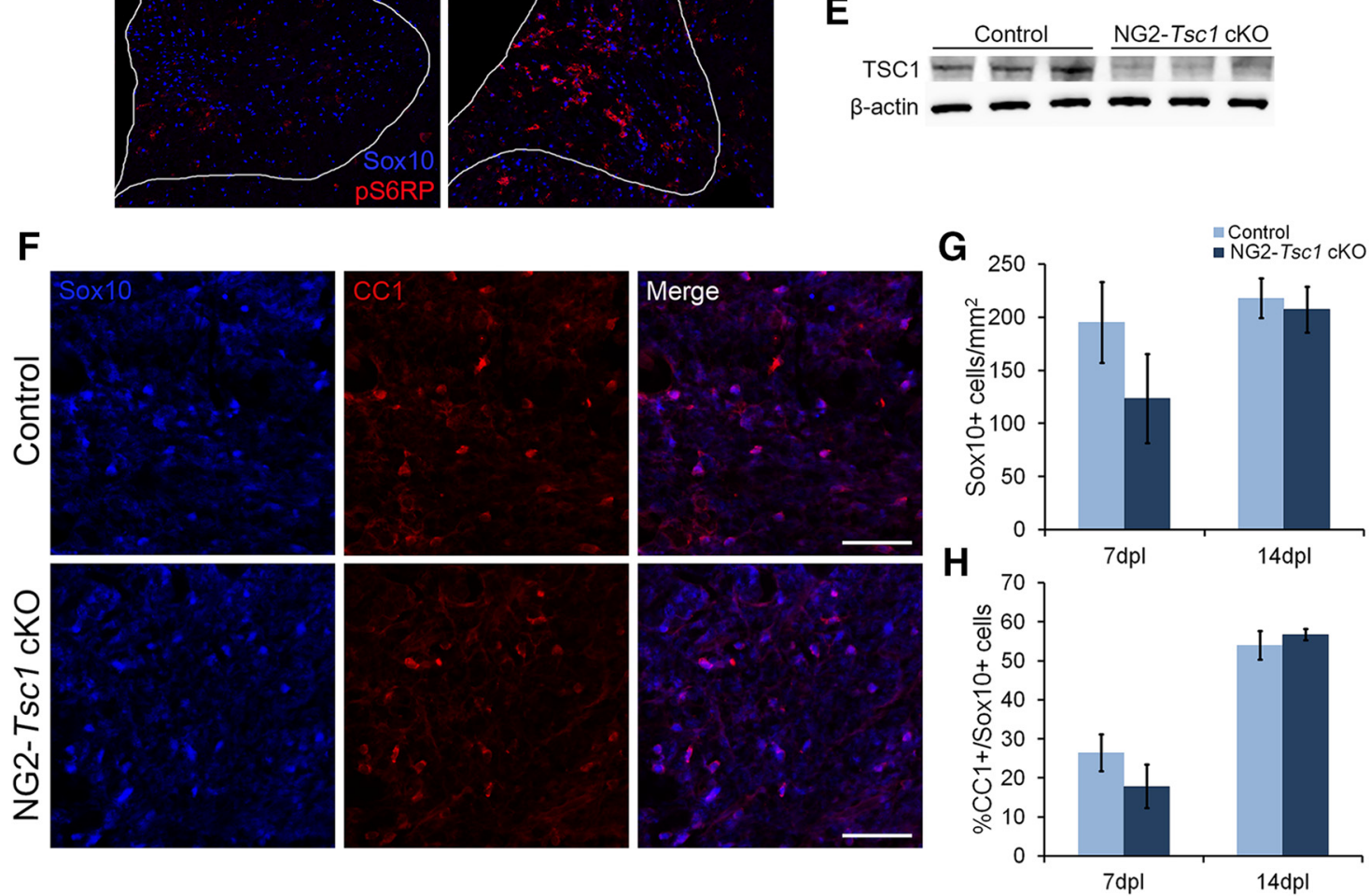

Figure 1. Tsc1 deletion from NG2 ${ }^{+}$cells increases mTORC1 signaling, but does not affect oligodendrocyte differentiation. $A$, Timeline of tamoxifen administration, lysolecithin injection, and sample collection. B, Expression of GFP reporter in NG2-Tsc1CK0 lesion at $14 \mathrm{dpl}$. Scale bar, $100 \mu \mathrm{m}$. C, Colabeling of Sox10, CC1, and GFP in $14 \mathrm{dpl}$ lesion of NG2-Tsc1cK0 animal. Scale bar, $10 \mu \mathrm{m}$. $D$, Representative images from control and NG2-Tsc1CK0 animals of pS6RP expression in Sox $10^{+}$cells at 14 dpl. Scale bar, $100 \mu \mathrm{m}$. E, Representative Western blot of TSC1 from control and NG2-Tsc1cK0 animals. F, Colabeling of Sox10 and CC1 at $14 \mathrm{dpl}$ in the lesions of control and NG2-Tsc1cK0 animals. Scale bar, $100 \mu \mathrm{m} . \mathbf{G}, \boldsymbol{H}$, Quantification of number of Sox $10^{+}$cells $/ \mathrm{mm}^{2}{ }^{2}(\boldsymbol{G})$ and percentage of Sox $10^{+}$cells that were also $\mathrm{CC}^{+}(\boldsymbol{H})$ within the lesion at 7 and $14 \mathrm{dpl}$. For $7 \mathrm{dpl}, n=5$ control, $n=3 \mathrm{NG2}$-Tsc $1 \mathrm{CKO}$; for $14 \mathrm{dpl}, n=4 /$ group. All values are expressed as \pm SEM.

the lesion borders but not in the necrotic lesion center. For g-ratio analysis, a minimum of 100 axons from two different areas of the lesion cross-section were analyzed from three animals per group. The resulting trend lines were then compared by linear regression analysis.

\section{Results}

Deletion of $T s c 1$ from adult OPCs increases mTORC1 signaling but does not affect oligodendrocyte differentiation Demyelination in the dorsal white matter of the murine spinal cord was induced by a single injection of lysolecithin. This method was chosen to study remyelination for several reasons: (1) because it does not elicit a systemic immune response, so the primary repair response of oligodendrocyte progenitors can be more easily examined; (2) the processes of demyelination and remyelination can be readily separated from one another; and (3) the kinetics of repair are well defined (Jeffery and Blakemore, 1995; Luo et al., 2014). Previous studies have shown that the lysolecithin-induced lesions are spontaneously remyelinated by resident $\mathrm{NG}_{2}{ }^{+} \mathrm{OPCs}$ that migrate into the lesion, proliferate, and differentiate into myelin-producing oligodendrocytes (Zawadzka et al., 2010). To study the function of TSC in oligodendrocyte progenitors during remyelination, $T s c 1$ was deleted from OPCs in adult mice before demyelination through a tamoxifen-inducible Cre recombinase expressed in $\mathrm{NG}_{2}{ }^{+}$cells (NG2-TsclcKO). The paradigm for tamoxifen injections, induction of the lysolecithin lesion, and tissue harvest is described in the Materials and Methods and shown in Figure $1 A$. 
We first confirmed successful recombination and functional disruption of the TSC1-TSC2 complex in the NG2-Tsc1cKO animals through GFP reporter expression, TSC1 protein expression, and the expression of phospho-S6 ribosomal protein (pS6RP), a downstream target of mTORC1/p70S6 kinase normally inhibited by TSC activity. GFP expression was observed throughout the lesion at $14 \mathrm{dpl}$ in NG2-Tsc1cKO animals, colabeling $86.1 \pm 5.3 \%$ of Sox $10^{+}$oligodendroglia and $86.5 \%$ of $\mathrm{CC}^{+}$mature oligodendrocytes (Fig. $1 B, C$ ). Moreover, the percentage of oligodendroglia expressing TSC1 in NG2-Tsc1cKO animals was $42.3 \pm 10.6 \%$ of controls. The percentage of Sox $10^{+}$ cells that expressed TSC1 was $30.3 \pm 7.6 \%$ in NG2-Tsc1cKO animals, down from $71.5 \pm 3.0 \%$ in control animals. TSC1 knockdown was confirmed by Western blot analysis; protein extracted from $\mathrm{NG}_{2}{ }^{+}$cells of the brain and spinal cords of unlesioned adult mice that underwent a comparable tamoxifen injection paradigm revealed that TSC1 protein expression was markedly reduced in NG2-Tsc1cKO animals compared with controls (Fig. 1E). Furthermore, Sox $10^{+}$oligodendroglia in NG2-Tsc1cKO animals had increased staining for $\mathrm{pS6RP}$, indicating increased mTORC1 activity (Fig. 1D). Together, these data suggest that Sox $10^{+}$cells were successfully recombined, with GFP reporter expression, loss of TSC1 and consequent disinhibition of mTORC1 signaling.

To determine whether the loss of Tsc1 in the adult OPCs in the NG2-Tsc1cKO animals altered OPC differentiation, we analyzed CC1 expression, a marker of mature oligodendrocytes (Fig. $1 F$ ). The total number of oligodendrocyte lineage cells, as assessed by expression of the transcription factor Sox 10, was not significantly different between control and NG2-Tsc1cKO animals at 7 and $14 \mathrm{dpl}$ (Fig. $1 G$ ). Because Tsc1 deletion was previously found to increase cell death in the oligodendrocyte lineage during development (Jiang et al., 2016), we assessed oligodendroglia expression of the apoptosis marker cleaved-caspase-3 at $14 \mathrm{dpl}$. Consistent with our findings on oligodendrocyte lineage cell number, we found no difference in the number of Sox $10^{+}$cells that expressed cleavedcaspase- 3 with loss of Tsc1 (control: $19.04 \pm 3.74$ cells/mm ${ }^{2}$, NG2TsclcKO: $15.88 \pm 3.08$ cells $\left./ \mathrm{mm}^{2}\right)$.

The percentage of Sox $10^{+}$oligodendroglia that were $\mathrm{CC} 1^{+}$ mature oligodendrocytes also did not vary between genotypes; $\sim 20 \%$ of oligodendroglia at $7 \mathrm{dpl}$ and $\sim 55 \%$ at $14 \mathrm{dpl}$ were $\mathrm{CC}^{+}{ }^{+}$in both the control and NG2-Tsc1cKO animals (Fig. $1 \mathrm{H}$ ). Therefore, there was no indication of altered oligodendrocyte differentiation with loss of Tsc1 in OPCs.

\section{Tsc1 deletion in NG2 ${ }^{+}$OPCs and pericytes has no effect on initial lesion size or monocyte activation}

NG2 is well known to be expressed in the adult OPCs that repair demyelinated lesions, but NG2 has also been described in pericytes and in some activated monocytes after injury (Kucharova et al., 2011). To determine whether the NG2-Cre induced recombination in these cell types in the lesions, we analyzed expression of the GFP reporter with Iba1, a marker of activated microglia and macrophages, and PDGFR $\beta$ to identify pericytes. We found no colocalization of GFP with Ibal (Fig. $2 A$ ), consistent with the finding that NG2 is expressed in activated monocytes and not in quiescent microglia in the unperturbed CNS. Because tamoxifen was administered before the lysolecithin lesion in our paradigm, none of the quiescent monocytes would be expected to have an active NG2 promoter and thus would escape recombination of the floxed Tsc1 allele. However, $54.6 \pm 4.37 \%$ of the PDGFR $\beta^{+}$ pericytes colocalized with GFP indicative of active Cre and recombination within these cells (Fig. 2B). Although Cre is active in pericytes within this model, the expression of TSC1 within peri-
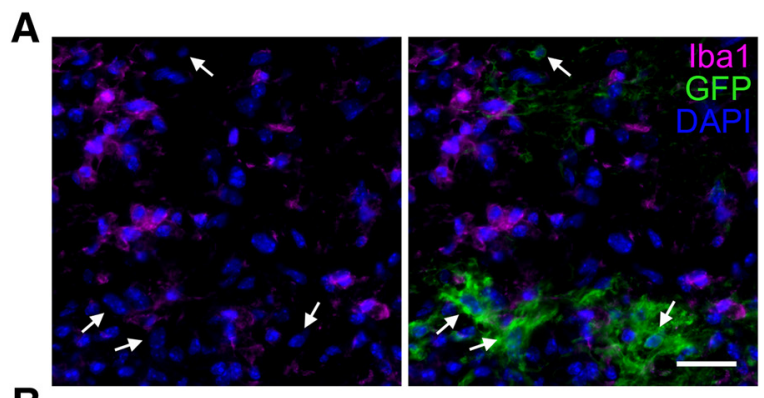

$\mathbf{B}$

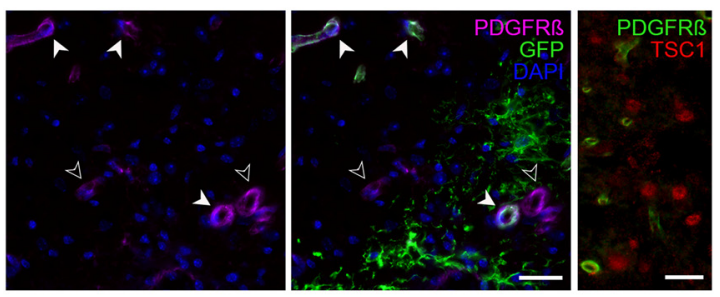

C

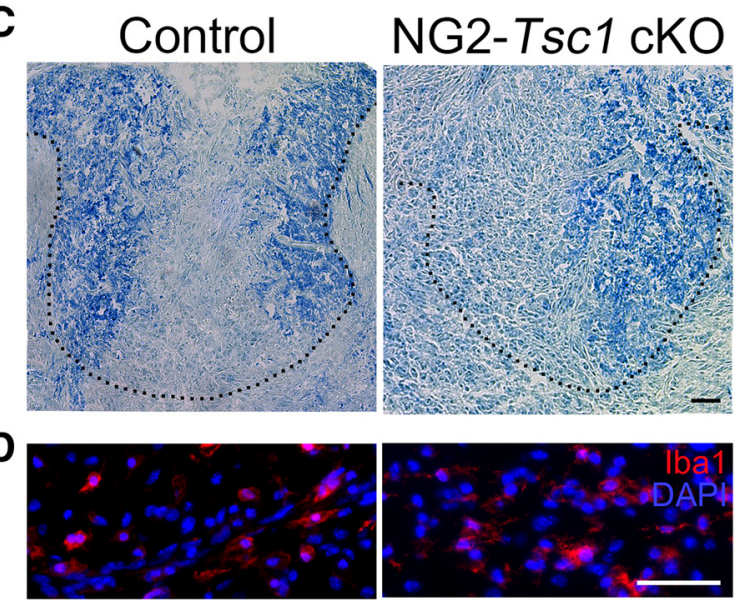

Figure 2. Initial lesion size and microgial/macrophage response are unchanged with Tsc1 deletion from $\mathrm{NG}_{2}{ }^{+}$cells. $\boldsymbol{A}$, Representative image of Iba1 staining and GFP reporter in the lesion of an NG2-Tsc1 cKO animal. Arrows indicate GFP ${ }^{+}$cells, all of which are negative for lba1. Scale bar, $50 \mu \mathrm{m}$. $\boldsymbol{B}$, Left two panels depict colocalization of pericyte marker PDGFR $\beta$ with GFP reporter in NG2-Tsc1cK0 lesion. Solid arrowheads identify colocalization; hollow arrowheads identify PDGFR $\beta^{+} /$GFP-cells. Rightmost panel shows low expression of TSC1 in PDGFR $\beta$ positive pericytes in control animals. Scale bars, $50 \mu \mathrm{m}$. C, Representative images from control and NG2-Tsc1cK0 animals of Luxol fast blue staining of the dorsal column at $7 \mathrm{dpl}$. Dotted line denotes borders of dorsal white matter. Scale bar, $100 \mu \mathrm{m}$. D, Immunostaining for lba1 at $7 \mathrm{dp}$ in control and NG2-Tsc1CKO animals. Scale bar, $100 \mu \mathrm{m}$.

cytes in control sections was found to be very low (Fig. 2B). Moreover, upon examination, the number of $\mathrm{CD}^{+}{ }^{+} \mathrm{T}$ cells and $\mathrm{Iba}^{+}$monocytes in unlesioned white matter did not differ between control and NG2-Tsc1cKO animals (data not shown). Given these data, it is not expected that deletion of TSC1 from pericytes would have a significant effect on the demyelination or remyelination of the lysolecithin lesion.

Because tamoxifen was administered before the injection of lysolecithin and because pericytes can regulate the blood-brain barrier, we sought to determine whether there were differences in initial lesion size or in number of microglia/macrophages within the demyelinated lesions. To address initial lesion size, we calculated total lesion volume by Luxol fast blue staining at $7 \mathrm{dpl}$, when lesion size is maximal before the onset of remyelination. Although there was some variability in lesion volume across all animals, there was no significant difference in lesion volume between groups at $7 \mathrm{dpl}$ (control: $0.86 \pm 0.3 \mathrm{~mm}^{3}$; NG2-TsclcKO: 
$0.60 \pm 0.23 \mathrm{~mm}^{3}$; Fig. 2C). Moreover, similar results were obtained when MBP staining was quantified in cross-sections taken at lesion peak at $7 \mathrm{dpl}$; there was no significant difference between controls and NG2-Tsc1 animals in the $\mathrm{MBP}^{-}$area (control: $0.44 \pm 0.06 \mathrm{~mm}^{2}$; NG2-Tsc1cKO: $0.40 \pm 0.17 \mathrm{~mm}^{2}$ ). We further analyzed Ibal staining within the lesion to assess potential differences in microglial/macrophage infiltration. There were no consistent changes in $\mathrm{Ibal}^{+}$cell number or intensity with genotype (Fig. 2D). These data support the conclusion that $T s c 1$ deletion in $\mathrm{NG}^{+}$cells before the lesion induction had no impact on the initial lesion size or on microglia/monocyte infiltration into the lesion.

\section{Tsc1 deletion in adult OPCs accelerates myelin protein expression during remyelination}

To address whether deletion of Tsc1 in adult OPCs affected remyelination, we first performed immunohistochemistry for MBP and MOG at 14 and $21 \mathrm{dpl}$ and quantified the percentage of the lesion that expressed MBP or MOG (Fig. 3A). Analysis revealed a main effect of genotype for both MBP and MOG with greater myelin protein expression in NG2-Tsc1cKO animals compared with controls across the two time points $\left(F_{(1,14)}=\right.$ $15.79, p=0.001$, ANOVA and $F_{(1,11)}=7.02, p=0.023$, ANOVA, respectively; Fig. $3 B$ ). Moreover, an increase in the percentage of the lesioned area expressing MBP was seen at $14 \mathrm{dpl}$ in the NG2TsclcKO (post hoc comparisons using the Sidak test indicated significant differences, adjusted $p=0.003$; Fig. $3 B$ ). Because nearly the entire lesioned area was $\mathrm{MBP}^{+}$at $14 \mathrm{dpl}$ in the NG2Tsc1cKO animals, the percentage of $\mathrm{MBP}^{+}$area increased only marginally from a mean of $90.4 \pm 1.8 \%$ at $14 \mathrm{dpl}$ to $95.5 \pm 3.6 \%$ at $21 \mathrm{dpl}$. Conversely, within the control lesions, MBP immunoreactivity increased from an average of $69.0 \pm 4.7 \%$ of lesioned area at $14 \mathrm{dpl}$ to $83.5 \pm 4.9 \%$ at $21 \mathrm{dpl}$ (Fig. $3 B$ ). Therefore, at $21 \mathrm{dpl}$, the difference in $\mathrm{MBP}^{+}$area was no longer statistically different between the NG2-Tsc1cKO animals and control animals because the lesions in both groups were nearly entirely $\mathrm{MBP}^{+}$. Because MOG is a late marker of mature oligodendrocytes and myelinogenesis, we expected MOG expression to occur in later stages of remyelination. Accordingly, the mean percentages of $\mathrm{MOG}^{+}$area at both $14 \mathrm{dpl}$ and $21 \mathrm{dpl}$ were markedly lower than the percentages of $\mathrm{MBP}^{+}$area in both groups. NG2TsclcKO animals had a trend toward a greater percentage of $\mathrm{MOG}^{+}$lesioned area, although this was not significantly different at either time point. However, between 14 and $21 \mathrm{dpl}$, the mean percentage of lesion that was $\mathrm{MOG}^{+}$increased significantly in NG2-Tsc1cKO animals (post hoc comparisons using the Sidak test indicated significant differences, adjusted $p=0.029$ ), but not in control animals, suggesting that MOG expression was increasing more rapidly in NG2-TsclcKO animals (Fig. 3B). Therefore, the lesions in NG2-Tsc1cKO animals expressed both MBP and MOG at a faster rate than the control lesions. Collectively, these data support increased remyelination in the NG2TsclcKO animals as early as $14 \mathrm{dpl}$.

\section{Accelerated remyelination with Tsc1 deletion from OPCs}

To further assess remyelination, we analyzed toluidine bluestained semithin cross-sections of the lesion at $21 \mathrm{dpl}$ (Fig. 4A). Cross-sections from both control and NG2-Tsc1cKO animals were ranked from least remyelinated (rank of 1) to most remyelinated. When comparing similar areas of the lesion, NG2TsclcKO animals consistently ranked higher than controls based on more remyelinated axons ( $p=0.012$, see Materials and Methods for description of statistical analysis; Fig. $4 B$ ). An animal that ranked in the bottom half of the rankings was $3 \times$ more likely to be a control animal than an NG2-Tsc1cKO animal. These data support increased remyelination with Tsc1 deletion.

The prior analyses demonstrated an increase in remyelination in the NG2-TsclcKO lesions. To determine whether the differences in early remyelination between control and NG2-Tsc1cKO animals also altered myelin thickness, we examined remyelinated areas of the lesion using EM (Fig. 4C). Although the area of remyelination was larger in the NG2-Tsc1cKO animals, there was no change in the percentage of myelinated axons within the remyelinated areas of control and NG2-Tsc1cKO animals; 20\% of axons were unmyelinated in both groups (control: $22.64 \pm$ 7.81\%, NG2-Tsc1cKO: $18.00 \pm 4.41 \%$ ). However, g-ratio analysis revealed that the myelinated axons in the NG2-Tsc1cKO animals had significantly lower g-ratios and thus thicker myelin (Fig. $4 D$ ). The g-ratio for each axon was plotted as a function of its axon diameter; linear regression analysis revealed that the trend line for the NG2-Tsc1cKO animals was significantly lower than that for the control animals, indicating thicker myelin across the range of axon diameters ( $y$-intercepts: control $=0.73$; NG2TsclcKO $=0.71 ; F_{(1,3554)}=137.75, p<0.0001$, linear regression analysis). The slopes of these lines were not significantly different, but they trended toward significance $\left(F_{(1,3553)}=3.57\right.$, $p=0.059$, linear regression analysis), suggesting that the effect of Tscldeletion on myelin thickness was not the same across all axon diameters. Indeed, when g-ratios were averaged across small-diameter $(<1.5 \mu \mathrm{m})$ or large-diameter $(\geq 1.5 \mu \mathrm{m})$ axons, there was a significant decrease in g-ratio in the NG2TsclcKO animals in the large-diameter axons (Holm-Sidak test, $p=0.019$ ), but not in the small-diameter axons (Fig. $4 E$ ).

To determine whether the increase in myelin thickness represented accelerated remyelination or sustained hypermyelination, we next quantified EM images of the lesions in control and NG2TsclcKO animals at $49 \mathrm{dpl}$, a time point when remyelination should be complete. At this time, the g-ratios of NG2-Tsc1cKO animals were no longer significantly smaller than controls (Fig. $4 F$ ), indicating that, by the culmination of remyelination, there were no differences in myelin thickness between groups.

\section{Deletion of Tsc1 from PLP ${ }^{+}$oligodendrocytes has no effect on oligodendrocyte number or myelin protein expression in remyelinating lesions}

Tscl deletion from $\mathrm{NG}_{2}{ }^{+} \mathrm{OPCs}$ resulted in an increased rate of remyelination without changing the number of mature oligodendrocytes. In addition to the presence of $\mathrm{NG}_{2}{ }^{+} \mathrm{OPCs}$ in the adult CNS, premyelinating oligodendrocytes are present in and around MS lesions, particularly in chronically demyelinated MS lesions. Therefore, we investigated whether Tscl deletion later in the lineage, once the cells have differentiated into premyelinating oligodendrocytes, would have the same beneficial effect as Tsc1 deletion from OPCs. To delete Tsc1 from differentiating oligodendrocytes, we injected tamoxifen into mice that expressed both the floxed Tsc1 allele and a tamoxifen-inducible Crerecombinase expressed from the PLP promoter (PLP-TsclcKO). Within the adult spinal cord, PLP is expressed only in differentiating oligodendrocytes, not in OPCs (Guo et al., 2009). To induce recombination in the largest number of premyelinating oligodendrocytes, we injected tamoxifen from 8-13 dpl, during the peak of lesion repair associated with oligodendrocyte differentiation but before significant remyelination has occurred (Fig. $5 \mathrm{~A}$ ). To confirm Tsc1 deletion, we assessed TSC1 protein expression in Sox $10^{+}$cells at $21 \mathrm{dpl}$ (Fig. 5B). TSC1 expression in Sox $10^{+}$ 
oligodendroglia in the PLP-Tsc1cKO animals was $38.9 \pm 10.8 \%$ of control levels, confirming Tsc1 knock-down (Fig. 5C). The percentage of Sox $10^{+}$cells that expressed TSC1 was $30.6 \pm 8.5 \%$ in PLPTsc1cKO animals, similar to what was seen in the NG2-Tsc1cKO animals. This contrasted with the $78.8 \pm 2.8 \%$ of Sox $10^{+}$cells that expressed TSC1 in control animals. TSC1 knock-down in animals expressing the PLP-Cre and the floxed-Tsc1 allele was further determined through Western blot analysis. $\mathrm{O}^{+}$cells were harvested from the spinal cords of adolescent control and PLP-Tsc1cKO animals that had received tamoxifen during development. Within the resulting protein lysates, TSC1 was markedly decreased in the PLP-TsclcKO animals (Fig. 5D), demonstrating effective recombination and $T s c 1$ deletion in PLP-Cre; Tsc1 ${ }^{\text {fllfl }}$ animals that received tamoxifen injections.

Because Tscl deletion occurs once the cells have initiated differentiation in the PLP-Tsc1cKO model, we expected no change in the number of mature oligodendrocytes between groups. As anticipated, neither the total number of Olig $2^{+}$ cells $\left(\sim 140\right.$ cells $\left./ \mathrm{mm}^{2}\right)$ nor the percentage of Olig $2^{+}$cells that was $\mathrm{CCl}^{+}(\sim 70 \%)$ differed between control and PLP-TsclcKO animals (Fig. $5 E, F$ ).

We then assessed remyelination through myelin protein expression at $21 \mathrm{dpl}$ (Fig. 5G). Both genotypes had significant remyelination, with MBP and MOG expression covering $>75 \%$ of the lesion area. There was no significant difference in the percentage of lesioned area that was positive for either myelin protein (Fig. 5H).

\section{Tsc1 deletion from differentiated oligodendrocytes reduces remyelination}

To further investigate alterations in remyelination, we ranked toluidine blue-stained semithin cross-sections from each lesion (Fig. 6A). Surprisingly, we found that control animals ranked higher than PLP-Tsc1cKO animals in the extent of remyelination. An animal from the top half of ranks was more than twice as likely to be a control animal than a PL-TsclcKO animal $(p=0.039$, see Materials and Methods for details on statistical analysis; Fig. $6 B)$. Therefore, Tscl deletion from differentiated oligodendrocytes had a negative effect on remyelination.

The reduction in remyelination in the PLP-TsclcKO animals was confirmed by ultrastructural EM analysis (Fig. 6C). When examining areas of remyelination, there was no change in the number of myelinated axons between control and PLP-Tsc1cKO animals (control: $14.1 \pm 2.8 \%$, PLP-Tsc1cKO: $21.4 \pm 1.0 \%$ ). However, the g-ratios of the PLP-Tsc1cKO animals were significantly larger than in control animals, indicating that the myelin sheaths on the remyelinated axons were thinner. Linear regression analysis of g-ratios plotted in relation to the corresponding axon diameter revealed that, whereas the slopes of the two trend lines were nearly identical (control: $0.049 \pm 0.0036$; PLP-Tsc1cKO: $0.049 \pm$ 0.0035), the $y$ intercept of the PLP-Tsc1cKO line was significantly higher than the control line (control: $0.69 \pm 0.005$; PLP-Tsc1cKO: $0.73 \pm 0.006 ; F_{(1,1928)}=106.69, p<0.0001$, linear regression anal- ysis). Therefore, myelin was consistently thinner in the PLPTsclcKO animals compared with controls across the range of axon diameters. To determine whether this effect on myelin thickness persisted, we also performed g-ratio analysis on electron micrographs of the lesion at $49 \mathrm{dpl}$ (Fig. 6D). At this time, no differences in myelin thickness were seen between control and PLP-Tsc1cKO animals, indicating that the hypomyelination observed at $21 \mathrm{dpl}$ was a transient effect of Tscl deletion from differentiated oligodendrocytes.

\section{Discussion}

Here, we show that disruption of TSC, an upstream inhibitor of mTORC1 signaling, has differential effects on remyelination depending on when in the oligodendrocyte lineage it is deleted. These findings are summarized in Figure 7. Deletion of Tsc1 from $\mathrm{NG}_{2}{ }^{+}$OPCs has no effect on OPC differentiation, but accelerates remyelination. NG2-Tsc1cKO animals have higher MBP expression early during remyelination, an increased rate of MOG accumulation, increased extent of remyelination throughout the lesion area, and smaller g-ratios for remyelinated axons compared with control animals. These data are indicative of an increase in both area of remyelination and thickness of myelin on remyelinated axons at $21 \mathrm{dpl}$. There were no changes in the initial lesion size or in the infiltration of monocytes/microglia into the lesions, supporting the conclusion that the increase in remyelination was due to loss of TSC1 in the oligodendrocytes. Because the increase in remyelination is seen without an increase in the number of mature oligodendrocytes, greater remyelination may be due to either an increase in the number of $\mathrm{CC}^{+}{ }^{+}$mature oligodendrocytes that contribute to remyelinating denuded axons or, alternatively, to an increase in the amount of myelin produced by each myelinating oligodendrocyte. Prior evidence suggests that mTOR signaling plays a role in the amount of myelin produced per oligodendrocyte. Hyperactivating the mTOR pathway through the constitutive activation of AKT causes hypermyelination without increasing the number of mature 
A
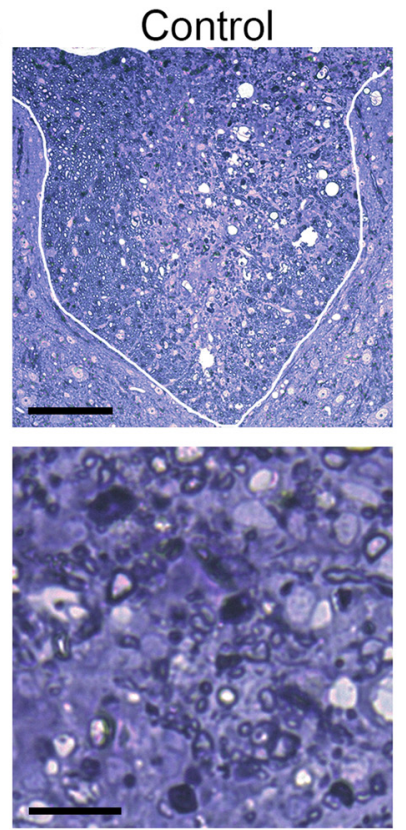

C
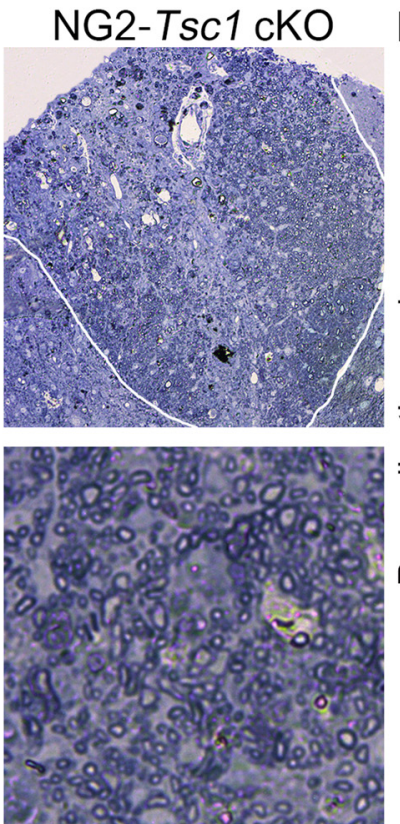

B

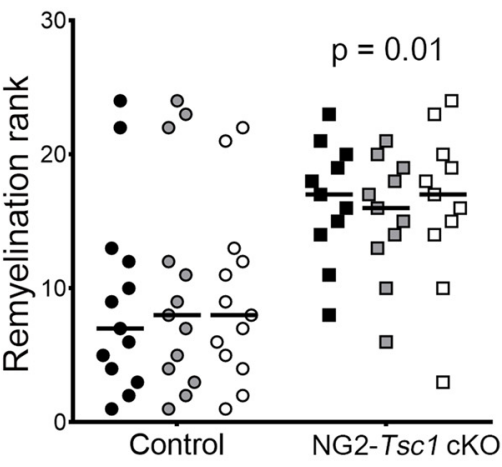

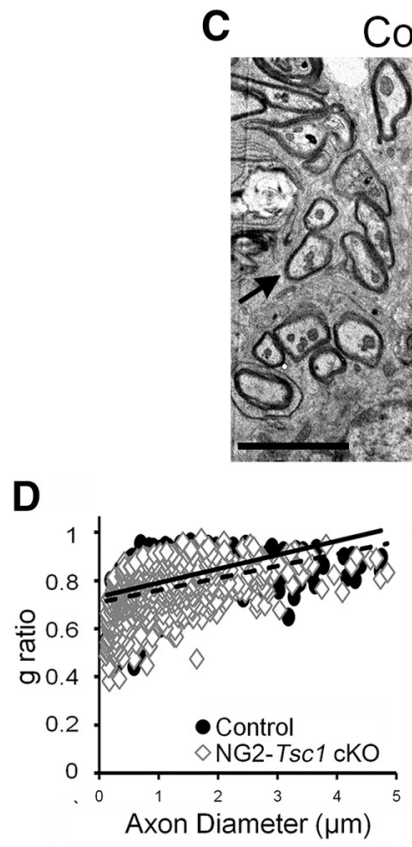

Control

NG2-Tsc1 cKO

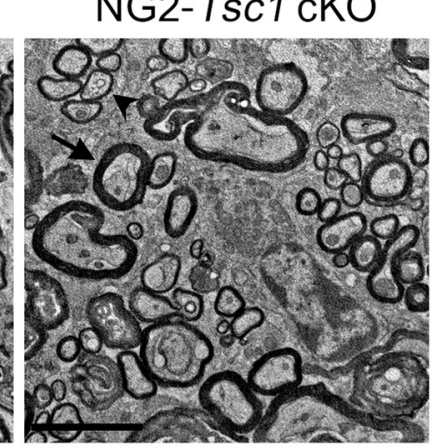

E

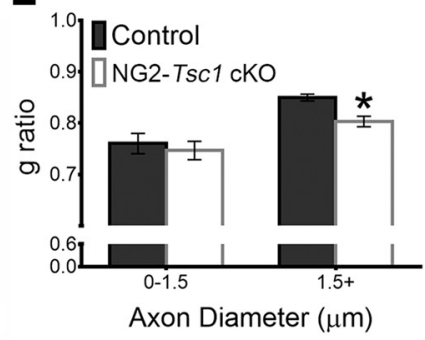

F

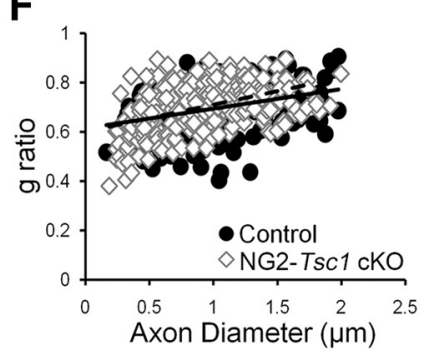

Figure 4. Remyelination is accelerated in NG2-Tsc1CKO animals. A, Low (top) and high (bottom) magnification images of toluidine blue-stained semithin sections of control and NG2-Tsc1cKO animals at $21 \mathrm{dpl}$. Low-magnification images capture the entire lesioned dorsal white matter; high-magnification images show remyelinating axons at the lesion border. Scale bars: top, $100 \mu \mathrm{m}$; bottom, $20 \mu \mathrm{m} . \boldsymbol{B}$, Rank analysis of toluidine blue images by three independent, blinded rankers with higher ranks indicating greater remyelination. Each symbol represents an independent section; two to three independent sections/animal were used in the ranking. Lines indicate median rank. $n=6$ control animals, $n=5 \mathrm{NG2}-T_{\mathrm{Sc}} 1 \mathrm{cKO}$ animals. C, Representative EMs from lesioned spinal cord of control and NG2-TSC 1 CKO animals at $21 \mathrm{dpl}$. Arrows and arrowheads denote axons of similar axon diameter representing axons of large $(\geq 1.5 \mu \mathrm{m})$ and small $(<1.5 \mu \mathrm{m})$ diameter, respectively. Scale bar, $4 \mu \mathrm{m}$. D, Quantification of myelin thickness through g-ratio analysis at $21 \mathrm{dpl}$. The g-ratios of individual axons were plotted in relation to their diameter. Control animals are represented by the solid trend line; NG2-TsC1CK0 animals by the dashed trend line. ${ }^{* * *} p<0.0001$, linear regression analysis. E, G-ratios were compared within small-diameter ( $\left.<1.5 \mu \mathrm{m}\right)$ and large-diameter $(\geq 1.5 \mu \mathrm{m})$ axons. ${ }^{*} p<0.05$, Holm-Sidak test. All values are expressed as \pm SEM. $F$, G-ratios were quantified in control and NG2-Tsc1cK0 animals at 49 dpl. Linear regression analysis revealed no significant differences between the two trend lines (control, solid trend line; NG2-Tsc1CK0, dashed trend line). At both time points, g-ratio measurements were performed on $>100$ axons from two different regions of the remyelinated lesion in each animal. $n=3 /$ group.

oligodendrocytes (Flores et al., 2008). Similarly, deletion of PTEN, an upstream inhibitor of mTOR signaling, results in no differences in remyelination despite a $>50 \%$ reduction in oligodendroglia number (Harrington et al., 2010). In both cases, the hypermyelination or normal remyelination, respectively, cannot be attributed solely to oligodendrocyte number and must therefore result from increased myelin production by oligodendrocytes.

Given that $T s c 1$ deletion from OPCs accelerates remyelination without affecting oligodendrocyte differentiation, we reasoned that $T s c 1$ deletion from differentiating premyelinating oligodendrocytes would have a similar effect. Therefore, we were surprised 
A
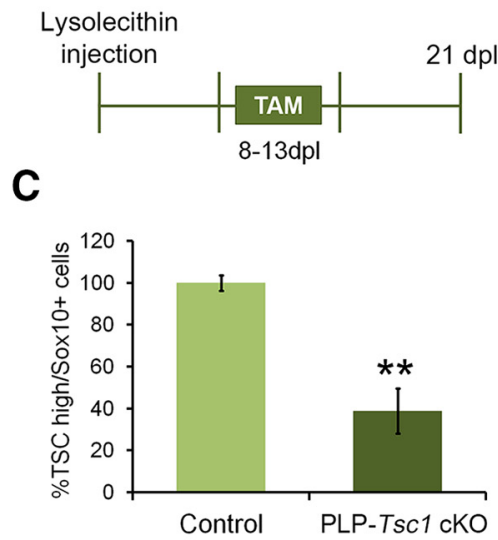

D

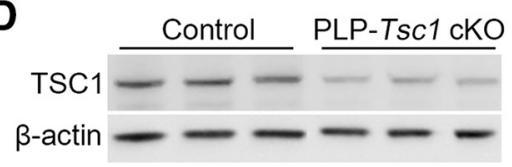

E

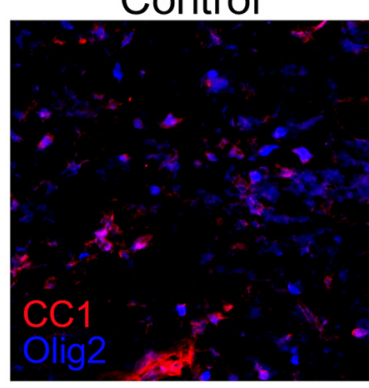

G
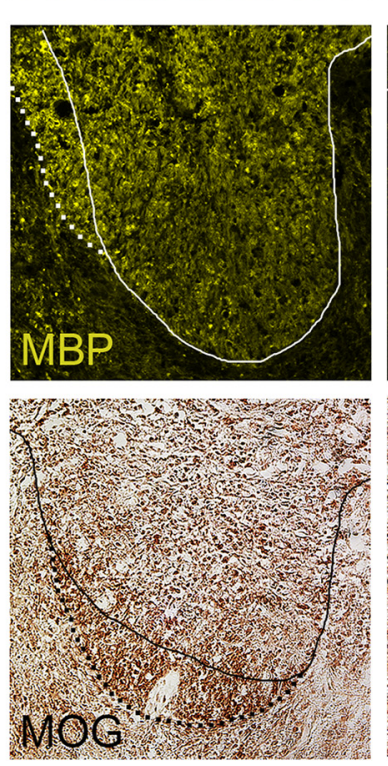

B
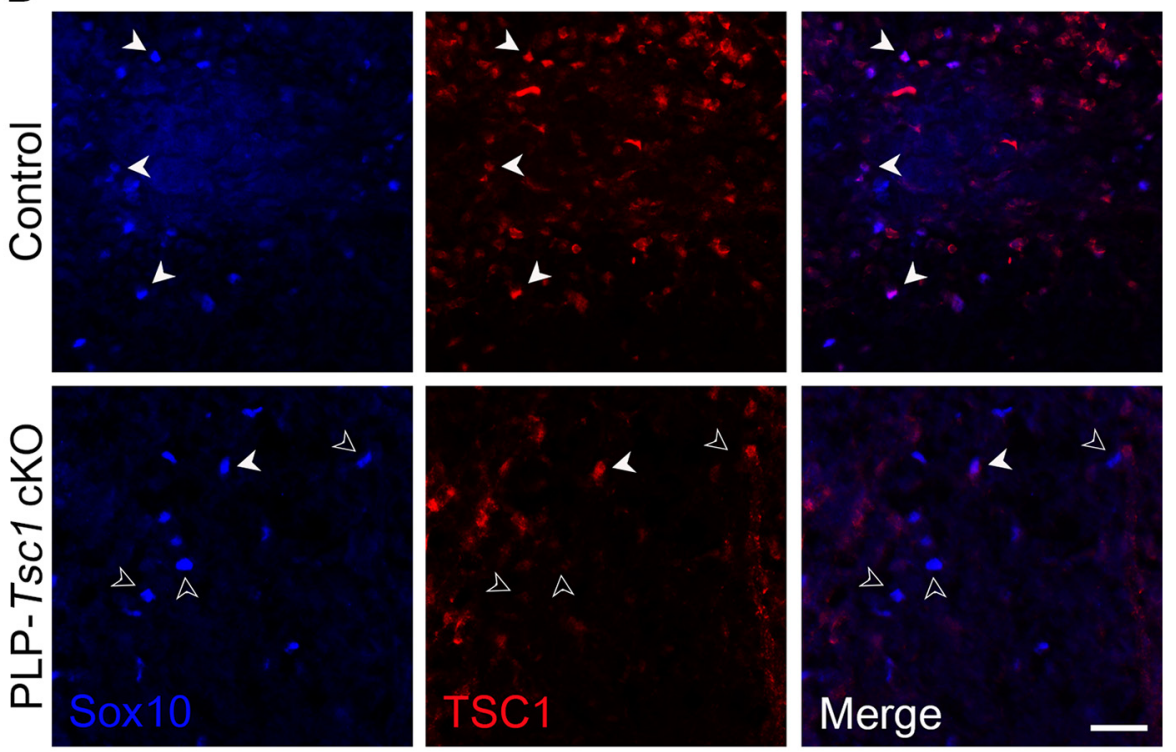

F
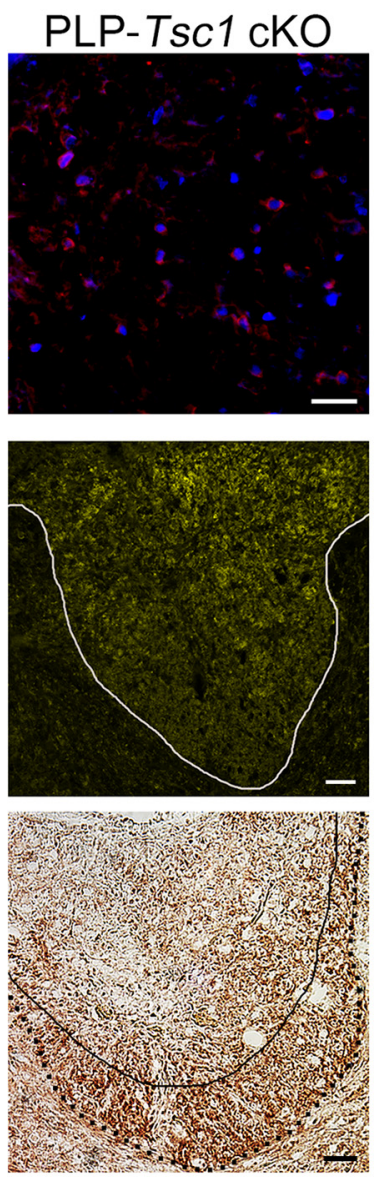

H
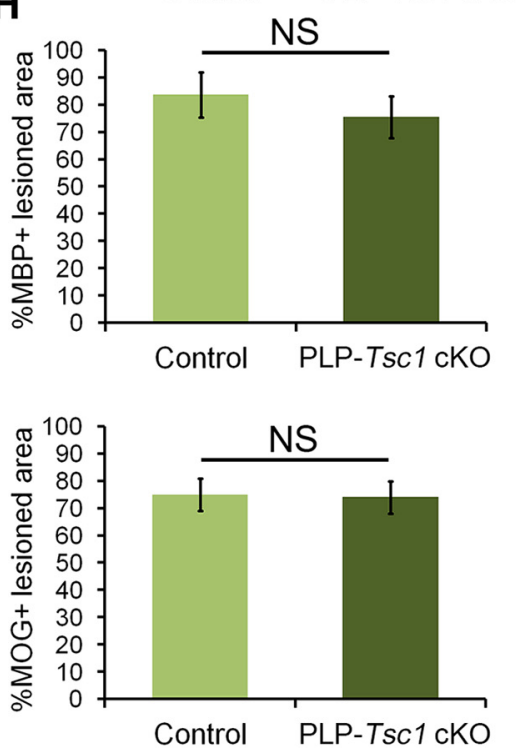

Figure 5. Tsc 7 deletion from differentiated oligodendrocytes has no effect on oligodendrocyte number or myelin protein expression. $A$, Timeline of lysolecithin injection, tamoxifen injections, and sample collection. $\boldsymbol{B}$, Representative images from control and PLP-Tsc1cK0 animals of TSC1 expression in Sox $10^{+}$cells. Solid arrowheads identify colocalization; hollow arrowheads identify Sox $10^{+}$ cells that are negative for TSC1. Scale bar, $50 \mu \mathrm{m}$. C, Quantification of the number of Sox $10^{+}$cells that have high TSC1 expression in PLP-TSC1CK0 animals compared with controls $\left(*^{* *} p<0.01\right)$. $n=4$ /group. D, Representative Western blots of TSC1 expression in $04^{+}$cells isolated from control and PLP-Tsc1 CKO animals. E, Colabeling of 0lig2 and CC 1 in lesions from control and PLP-Tsc 1 CKO animals. Scale bar, $50 \mu \mathrm{m}$. $\boldsymbol{F}$, Quantification of the total number of Olig2 ${ }^{+}$cells and $\mathrm{CC}^{+} / 0$ lig2 ${ }^{+}$cells. $n=4 /$ group. $\mathbf{G}$, Immunostaining for myelin proteins MBP (top) and MOG (bottom). Solid lines denote lesion boundaries; dotted lines denote boundaries of dorsal white matter. Scale bars, $100 \mu \mathrm{m}$. $\mathbf{H}$, Quantification of the percentage of lesioned area that was MBP ${ }^{+}$or MOG ${ }^{+}$at $21 \mathrm{dpl}$ in control and PLP-Tsc1CKO animals. $n=5$ control, 4 PLP-Tsc1CKO. NS, Not significant. All values are expressed as \pm SEM. 
A
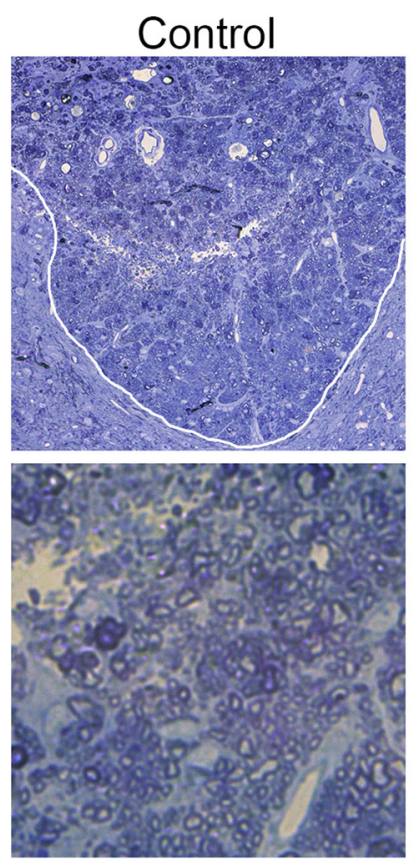

C

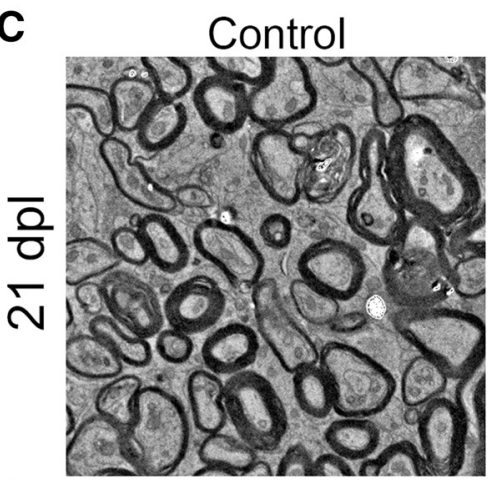

D

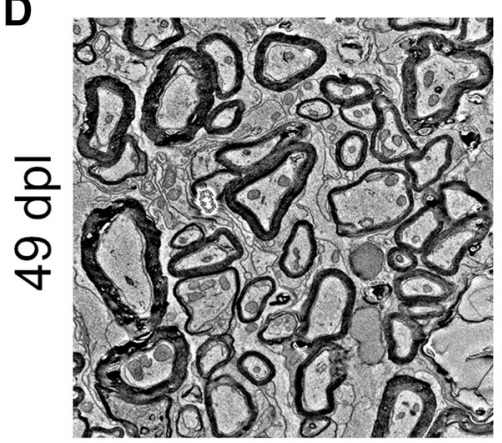

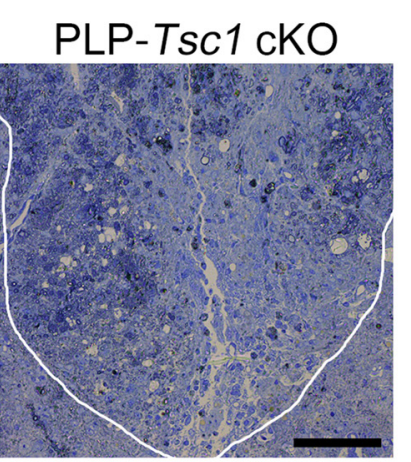

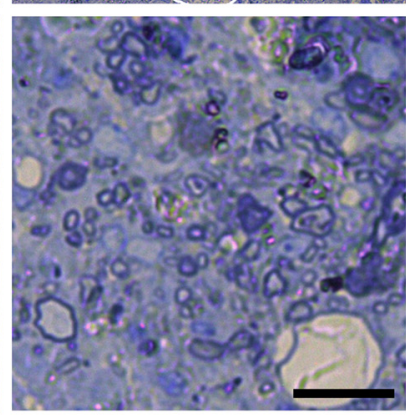

PLP-TSC1 cKO
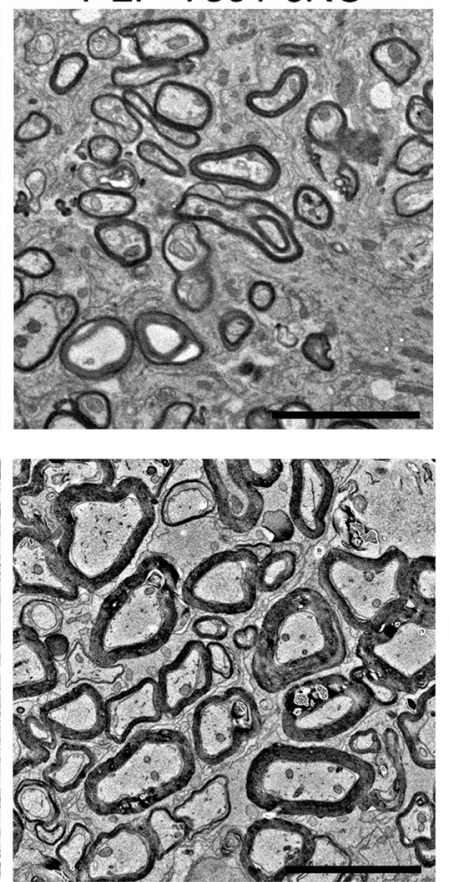

B

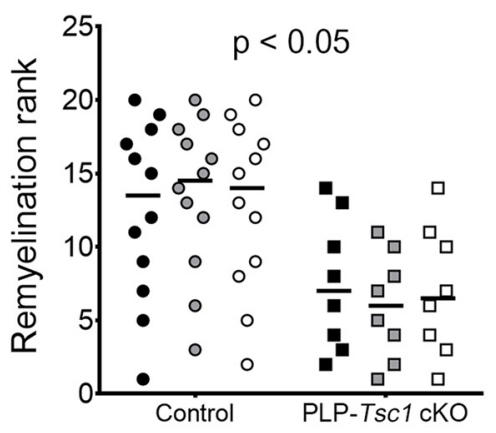

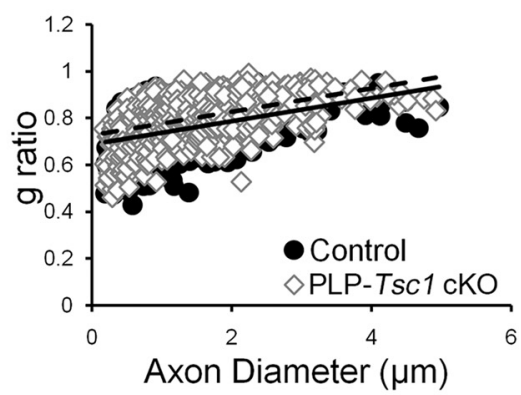

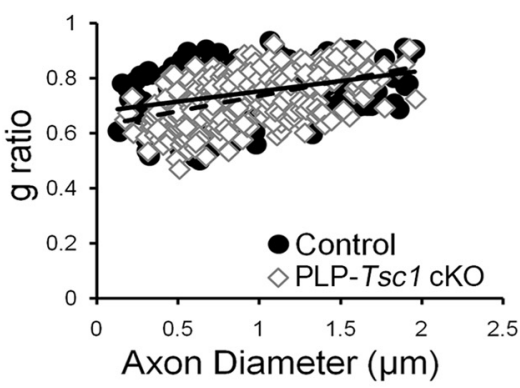

Figure 6. Remyelination is slower in PLP-TSC1 CKO animals. A, Representative images of toluidine blue-stained semithin sections from $21 \mathrm{dpl}$ control and PLP-Tsc 1 cKO animals at low (top) and high (bottom) magnification. Low-magnification images capture the entire lesioned dorsal white matter; high-magnification images show remyelinating axons at the lesion border. Scale bars: top, $100 \mu \mathrm{m}$; bottom, $20 \mu \mathrm{m}$. B, Rank analysis of remyelination in toluidine blue images with the least remyelinated section receiving a rank of 1 . Each symbol represents an individual section; two independent sections were analyzed per animal. Each color represents a different ranker. Lines indicate median rank. $n=6$ control animals, $n=4$ PLP-Tsc 7 KKO animals. C, $\boldsymbol{D}$, Representative EMs from control and PLP-Tsc1cK0 animals and g-ratio scatterplots at $21 \mathrm{dpl}(\boldsymbol{C})$ and $49 \mathrm{dpl}(\boldsymbol{D})$. Scale bar, $4 \mu \mathrm{m}$. Control animals are represented by the solid trend line; PLP-Tsc1cK0 animals by the dashed trend line. Linear regression analysis revealed a significantly higher $y$-intercept for the PLP-Tsc1CKO trend line at $21 \mathrm{dpl,}{ }^{* * *} p<0.0001$. No differences were seen between control and PLP-Tsc7cK0 animals in g-ratios across axon diameters at $49 \mathrm{dpl}$.

to find that deletion of $T s c 1$ from $\mathrm{PLP}^{+}$oligodendrocytes had the opposite effect and slowed remyelination. Although there was no obvious change in myelin protein expression, both the remyelination rank and the thickness of the myelin on the remyelinated axons were less than controls at $21 \mathrm{dpl}$. It is unclear why deletion of $T s c 1$ from different stages of the oligodendrocyte lineage has differential effects. One possible explanation lies in the modula- tion of mTORC2 by TSC and differential function of mTORC1 and mTORC2 signaling in oligodendroglial maturation. Although TSC has classically been described as an upstream inhibitor of mTORC1, it is also an activator of mTORC2, albeit through unknown mechanisms (Huang et al., 2008). It is plausible that $\mathrm{mTORC} 1$ and $\mathrm{mTORC} 2$ play interconnected but distinct roles in the regulation of OPC differentiation and myelina- 
tion. Recent studies have suggested that mTORC1 has a larger role during developmental myelination; inhibition of mTORC1 signaling through the deletion of Rptor results in reduced oligodendrocyte differentiation and myelination during development, similar to the phenotype seen with mTOR deletion (Bercury et al., 2014; Lebrun-Julien et al., 2014). The function of mTORC2 is less clear. Deletion of Rictor and consequent abrogation of mTORC2 signaling has no effect on developmental myelination, although there is an increase in mature oligodendrocytes (Bercury et al., 2014). This suggests the possibility that a greater number of oligodendrocytes are needed to produce normal levels of myelin when mTORC2 signaling is disrupted. Moreover, mTORC2 phosphorylates AKT at Ser473, a site targeted in the constitutively active AKT mutant that results in developmental hypermyelination (Flores et al., 2008). Together, these studies suggest that mTORC2 signaling may have a subtle but important role in the regulation of myelin production by oligodendrocytes.

It is plausible that $T s c 1$ deletion in OPCs and the consequent increase in mTORC1 signaling may promote $\mathrm{CC}^{+}$ oligodendrocyte ensheathment and myelination of axons. In vitro studies indicate that mTOR is important, not only in the differentiation of OPCs to oligodendrocytes, but also in the maturation of immature oligodendrocytes to mature oligodendrocytes that express myelin proteins (Tyler et al., 2009; Guardiola-Diaz et al., 2012). Therefore, even though Tscl deletion from OPCs has no effect on the number of $\mathrm{CC}^{+}$oligodendrocytes, it might be important for the full maturation of these cells. Conversely, when Tsc1 is deleted from $\mathrm{PLP}^{+}$oligodendrocytes, the deletion may happen too late to influence the later stages of oligodendrocyte maturation, rendering the increase in mTORC1 signaling inconsequential. Concurrently, the acute reduction in mTORC2/Akt signaling at the onset of remyelination in our paradigm may result in reduced myelin production by these cells, accounting for the reduction in remyelination seen in PLP-Tsc1cKO animals. Although mTORC2 signaling would also be reduced in the NG2-Tsc1cKO animals, the increase in mTORC1 signaling may compensate by increasing the number of existing $\mathrm{CC}^{+}$oligodendrocytes that initiate remyelination of the denuded axons.

Our finding that OPC disruption of TSC, an upstream inhibitor of mTORC1, accelerates and enhances remyelination is in agreement with previous studies showing that systemic inhibition of mTOR signaling through rapamycin treatment inhibits remyelination in the cuprizone model (Sachs et al., 2014). Both of these studies implicate mTOR signaling in remyelination: in our current study, we show that deletion of an upstream inhibitor of mTORC1 accelerates remyelination; in the prior study, mTOR was inhibited and thus caused delayed remyelination. However, in disagreement with our findings, another study found that deletion of the upstream inhibitor PTEN does not affect remyelination (Harrington et al., 2010). It should be noted that PTEN was deleted during development in this latter study and resulted in hypermyelination throughout the CNS. It is unclear what effect developmental hypermyelination had on the ability of OPCs to respond to and repair a demyelinated lesion. Indeed, PTEN deletion markedly reduced adult OPC proliferation and thus the number of oligodendroglia during remyelination (Harrington et al., 2010). Therefore, although remyelination was not increased with PTEN deletion, repair mechanisms may have been hindered by altered environmental cues from the surrounding hypermyelinated white matter or by intrinsic changes within OPCs due to developmental PTEN deletion.

We have shown that deletion of Tsc1 from adult OPCs is beneficial to remyelination. However, others have shown that deletion of Tsc1 or Tsc2 from OPCs during development is 
detrimental, resulting in hypomyelination as a consequence of reduced oligodendroglial number (Carson et al., 2015; Jiang et al., 2016). The most likely explanation for why deletion of a Tsc gene from OPCs results in cell death or a lineage shift during development but not during remyelination is that adult OPCs are a distinctly different population from their developmental counterparts. A higher percentage of adult OPCs in vitro express $\mathrm{O} 4$, an antigen associated with late progenitors (Wolswijk and Noble, 1989). Adult OPCs are also more morphologically complex than developmental OPCs and differentiate more readily in culture (Wolswijk and Noble, 1989; Lin et al., 2009). Finally, microarray analysis has revealed that adult and developmental OPCs have distinct gene profiles, with adult OPCs expressing higher levels of myelin gene transcripts (Moyon et al., 2015). Together, these data suggest that adult OPCs are more mature than their developmental counterparts. Therefore, it is probable that $T s c 1$ deletion in $\mathrm{NG}_{2}{ }^{+}$adult OPCs before a demyelination insult results in recombination in cells that are at a later stage in the lineage than those targeted by the Olig1-Cre or Olig2-Cre during development. These more mature progenitors may be less likely to shift lineage identity and differentiate into astrocytes and may

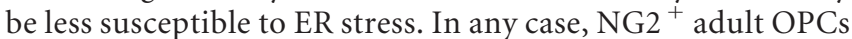
are clearly a distinct population from the OPCs seen in development and may simply respond differently to disruption of the TSC1-TSC2 complex, a conclusion supported by our findings that Tsc1 deletion from adult OPCs has no effect on cell death or oligodendroglia number and ultimately increases remyelination.

The findings presented here suggest a complex role for Tsc1 in remyelination. This emphasizes the importance of carefully considered therapeutics to enhance remyelination in diseases such as MS because what is beneficial to oligodendroglial development at one stage may be detrimental in another stage. The mTOR signaling pathway consistently appears to be an attractive target to enhance remyelination. It was shown previously to be essential for proper developmental myelination and for remyelination during active cuprizone treatment (Bercury et al., 2014; Sachs et al., 2014; Wahl et al., 2014). Previous studies have shown that promoting PI3K/Akt/mTOR signaling enhances developmental myelination. Here, we have presented the first evidence that disruption of the mTORC1 inhibitor TSC accelerates remyelination.

\section{References}

Bercury KK, Dai J, Sachs HH, Ahrendsen JT, Wood TL, Macklin WB (2014) Conditional ablation of raptor or rictor has differential impact on oligodendrocyte differentiation and CNS myelination. J Neurosci 34:44664480. CrossRef Medline

Carson RP, Kelm ND, West KL, Does MD, Fu C, Weaver G, McBrier E, Parker B, Grier MD, Ess KC (2015) Hypomyelination following deletion of Tsc2 in oligodendrocyte precursors. Ann Clin Transl Neurol 2:10411054. CrossRef Medline

Daniel WW (1990) Friedman two-way analysis of variance by ranks. In: Applied nonparametric statistics, pp 262-274. Boston: PWS-Kent.

Flores AI, Narayanan SP, Morse EN, Shick HE, Yin X, Kidd G, Avila RL, Kirschner DA, Macklin WB (2008) Constitutively active Akt induces enhanced myelination in the CNS. J Neurosci 28:7174-7183. CrossRef Medline

Goldschmidt T, Antel J, König FB, Brück W, Kuhlmann T (2009) Remyelination capacity of the MS brain decreases with disease chronicity. Neurology 72:1914-1921. CrossRef Medline

Griffiths I, Klugmann M, Anderson T, Yool D, Thomson C, Schwab MH, Schneider A, Zimmermann F, McCulloch M, Nadon N, Nave KA (1998) Axonal swellings and degeneration in mice lacking the major proteolipid of myelin. Science 280:1610-1613. CrossRef Medline
Guardiola-Diaz HM, Ishii A, Bansal R (2012) Erk1/2 MAPK and mTOR signaling sequentially regulates progression through distinct stages of oligodendrocyte differentiation. Glia 60:476-486. CrossRef Medline

Guo F, Ma J, McCauley E, Bannerman P, Pleasure D (2009) Early postnatal proteolipid promoter-expressing progenitors produce multilineage cells in vivo. J Neurosci 29:7256-7270. CrossRef Medline

Harrington EP, Zhao C, Fancy SP, Kaing S, Franklin RJ, Rowitch DH (2010) Oligodendrocyte PTEN is required for myelin and axonal integrity, not remyelination. Ann Neurol 68:703-716. CrossRef Medline

Huang J, Dibble CC, Matsuzaki M, Manning BD (2008) The TSC1-TSC2 complex is required for proper activation of mTOR complex 2. Mol Cell Biol 28:4104-4115. CrossRef Medline

Inoki K, Li Y, Xu T, Guan KL (2003) Rheb GTPase is a direct target of TSC2 GAP activity and regulates mTOR signaling. Genes Dev 17:1829_ 1834. CrossRef Medline

Ipe D (1987) Performing the Friedman test and the associated multiple comparison test using PROC GLM. In: Twelfth Annual SAS Users Group International Conference, pp 1146-1148. Cary, NC: SAS Institute Inc. Available at http://www.sascommunity.org/sugi/SUGI87/Sugi-12-209\%20Ipe.pdf.

Jeffery ND, Blakemore WF (1995) Remyelination of mouse spinal cord axons demyelinated by local injection of lysolecithin. J Neurocytol 24:775781. CrossRef Medline

Jiang M, Liu L, He X, Wang H, Lin W, Wang H, Yoon SO, Wood TL, Lu QR (2016) Regulation of PERK-eIF2alpha signalling by tuberous sclerosis complex-1 controls homoeostasis and survival of myelinating oligodendrocytes. Nat Commun 7:12185. CrossRef Medline

Keough MB, Yong VW (2013) Remyelination therapy for multiple sclerosis. Neurotherapeutics 10:44-54. CrossRef Medline

Kucharova K, Chang Y, Boor A, Yong VW, Stallcup WB (2011) Reduced inflammation accompanies diminished myelin damage and repair in the NG2 null mouse spinal cord. J Neuroinflammation 8:158. CrossRef Medline

Lappe-Siefke C, Goebbels S, Gravel M, Nicksch E, Lee J, Braun PE, Griffiths IR, Nave KA (2003) Disruption of Cnp1 uncouples oligodendroglial functions in axonal support and myelination. Nat Genet 33:366-374. CrossRef Medline

Lebrun-Julien F, Bachmann L, Norrmén C, Trötzmüller M, Köfeler H, Rüegg MA, Hall MN, Suter U (2014) Balanced mTORC1 activity in oligodendrocytes is required for accurate CNS myelination. J Neurosci 34:84328448. CrossRef Medline

Lin G, Mela A, Guilfoyle EM, Goldman JE (2009) Neonatal and adult $\mathrm{O} 4(+)$ oligodendrocyte lineage cells display different growth factor responses and different gene expression patterns. J Neurosci Res 87:33903402. CrossRef Medline

Luo F, Burke K, Kantor C, Miller RH, Yang Y (2014) Cyclin-dependent kinase 5 mediates adult OPC maturation and myelin repair through modulation of Akt and GsK-3 beta signaling. J Neurosci 34:10415-10429. CrossRef Medline

Moyon S, Dubessy AL, Aigrot MS, Trotter M, Huang JK, Dauphinot L, Potier MC, Kerninon C, Melik Parsadaniantz S, Franklin RJ, Lubetzki C (2015) Demyelination causes adult CNS progenitors to revert to an immature state and express immune cues that support their migration. J Neurosci 35:4-20. CrossRef Medline

Narayanan SP, Flores AI, Wang F, Macklin WB (2009) Akt signals through the mammalian target of rapamycin pathway to regulate CNS myelination. J Neurosci 29:6860-6870. CrossRef Medline

Patrikios P, Stadelmann C, Kutzelnigg A, Rauschka H, Schmidbauer M, Laursen H, Sorensen PS, Brück W, Lucchinetti C, Lassmann H (2006) Remyelination is extensive in a subset of multiple sclerosis patients. Brain 129:3165-3172. CrossRef Medline

Sachs HH, Bercury KK, Popescu DC, Narayanan SP, Macklin WB (2014) A new model of cuprizone-mediated demyelination/remyelination. ASN Neuro 6.

Shields SA, Gilson JM, Blakemore WF, Franklin RJ (1999) Remyelination occurs as extensively but more slowly in old rats compared to young rats following gliotoxin-induced CNS demyelination. Glia 28:77-83. CrossRef Medline

Tyler WA, Gangoli N, Gokina P, Kim HA, Covey M, Levison SW, Wood TL (2009) Activation of the mammalian target of rapamycin (mTOR) is essential for oligodendrocyte differentiation. J Neurosci 29:6367-6378. CrossRef Medline 
van Slegtenhorst M, Nellist M, Nagelkerken B, Cheadle J, Snell R, van den Ouweland A, Reuser A, Sampson J, Halley D, van der Sluijs P (1998) Interaction between hamartin and tuberin, the TSC1 and TSC2 gene products. Hum Mol Genet 7:1053-1057. CrossRef Medline

Wahl SE, McLane LE, Bercury KK, Macklin WB, Wood TL (2014) Mammalian target of rapamycin promotes oligodendrocyte differentiation, initiation and extent of CNS myelination. J Neurosci 34:4453-4465. CrossRef Medline

Wolswijk G, Noble M (1989) Identification of an adult-specific glial progenitor cell. Development 105:387-400. Medline

Zawadzka M, Rivers LE, Fancy SP, Zhao C, Tripathi R, Jamen F, Young K, Goncharevich A, Pohl H, Rizzi M, Rowitch DH, Kessaris N, Suter U, Richardson WD, Franklin RJ (2010) CNS-resident glial progenitor/ stem cells produce Schwann cells as well as oligodendrocytes during repair of CNS demyelination. Cell Stem Cell 6:578-590. CrossRef Medline

Zeger SL, Liang KY (1986) Longitudinal data analysis for discrete and continuous outcomes. Biometrics 42:121-130. CrossRef Medline

Zhang Y, Gao X, Saucedo LJ, Ru B, Edgar BA, Pan D (2003) Rheb is a direct target of the tuberous sclerosis tumour suppressor proteins. Nat Cell Biol 5:578-581. CrossRef Medline

Zou Y, Jiang W, Wang J, Li Z, Zhang J, Bu J, Zou J, Zhou L, Yu S, Cui Y, Yang W, Luo L, Lu QR, Liu Y, Chen M, Worley PF, Xiao B (2014) Oligodendrocyte precursor cell-intrinsic effect of Rhebl controls differentiation and mediates mTORC1-dependent myelination in brain. J Neurosci 34: 15764-15778. CrossRef Medline 